\title{
Ultrafast Nonadiabatic Fragmentation Dynamics of Doubly Charged Uracil in a Gas Phase
}

\author{
P. López-Tarifa, ${ }^{1}$ M.-A. Hervé du Penhoat, ${ }^{2}$ R. Vuilleumier, ${ }^{3}$ M.-P. Gaigeot, ${ }^{4,5}$ I. Tavernelli, ${ }^{6}$ A. Le Padellec, ${ }^{7,8}$ \\ J.-P. Champeaux, ${ }^{9,10}$ M. Alcamí, ${ }^{1}$ P. Moretto-Capelle, ${ }^{9,10}$ F. Martín, ${ }^{1,11, *}$ and M.-F. Politis ${ }^{4}$ \\ ${ }^{1}$ Departamento de Química, Módulo 13, Universidad Autónoma de Madrid, 28049 Madrid, Spain \\ ${ }^{2}$ IMPMC UMR-CNRS 7590, Université Pierre et Marie Curie, 4 place Jussieu, 75005 Paris, France \\ ${ }^{3}$ Ecole Normale Superieure, Departement de Chimie, UMR 8640 CNRS-ENS-UPMC, 75005 Paris, France \\ ${ }^{4} L A M B E$, UMR-CNRS 8587, Université d'Evry val d'Essonne, 91025 Evry, France \\ ${ }^{5}$ Institut Universitaire de France, 103 Blvd St Michel, 75005 Paris, France \\ ${ }^{6}$ Ecole Polytechnique Fédérale de Lausanne, 1015 Lausanne, Switzerland \\ ${ }^{7}$ CESR, Université de Toulouse, UPS, 31028 Toulouse Cedex 9, France \\ ${ }^{8}$ CNRS, UMR5187, F-31028 Toulouse, France \\ ${ }^{9}$ Laboratoire Collisions, Agrégats, Réactivité, Université de Toulouse, UPS, 31062 Toulouse, France \\ ${ }^{10}$ CNRS, UMR 5589, F-31062 Toulouse, France \\ ${ }^{11}$ Instituto Madrileño de Estudios Avanzados en Nanociencia (IMDEA-Nanociencia), Cantoblanco, 28049 Madrid, Spain
} (Received 6 October 2010; published 6 July 2011)

\begin{abstract}
A combination of time-dependent density functional theory and Born-Oppenheimer molecular dynamics methods is used to investigate fragmentation of doubly charged gas-phase uracil in collisions with $100 \mathrm{keV}$ protons. The results are in good agreement with ion-ion coincidence measurements. Orbitals of similar energy and/or localized in similar bonds lead to very different fragmentation patterns, thus showing the importance of intramolecular chemical environment. In general, the observed fragments do not correspond to the energetically most favorable dissociation path, which is due to dynamical effects occurring in the first few femtoseconds after electron removal.
\end{abstract}

DOI: 10.1103/PhysRevLett.107.023202

PACS numbers: $34.10 .+\mathrm{x}, 34.50 .-\mathrm{s}$

High-frequency electromagnetic radiation, energetic ions, and electrons can induce chemical changes that are lethal for living systems. For this reason, such radiation sources are often used in cancer therapies, which aim at damaging the DNA of malignant cells. In this field, the use of swift highly charged ions is very promising due to their ability to deposit energy and induce cellular death in very localized areas of deep tumors (a consequence of the wellknown localization of the Bragg peak [1]). These ions can produce DNA damage either directly, through ionization and excitation [1,2], or indirectly, through chemical reactions with the species produced in the aqueous environment. The early stages of damage, which occur during the first few femtoseconds after irradiation and lead to fragmentation of the biomolecule, are far from being understood. To unravel the mechanisms at this early stage, physicists have performed numerous experiments in gas phase (see, e.g., [3-5]), in which swift charged ionic projectiles impinge on DNA or RNA bases, sugars, nucleosides, or even biomolecular clusters. In contrast with experiments performed in solution or directly on living systems, gas-phase experiments provide direct and precise information on single collision events. Thus they allow one to unambiguously identify fragmentation channels associated with a given biomolecule and not with the environment. Such detailed information can be achieved by combining techniques that are state of the art in gas-phase chemistry, e.g., high resolution mass spectrometry, and in collision physics, such as multicoincidence detection techniques that provide the correlation between different charged fragments as well as their relative kinetic energies and momenta.

Fragmentation results from relaxation of the excess electronic energy associated with vacancies created in the different electronic shells of the molecule. Since, in these collisions, electrons can be removed from many of these shells, experiments cannot tell us how fragmentation depends (i) on the shape or energy of the molecular orbital (MO) in which the electron vacancies are created and (ii) on the intramolecular environment, i.e., on the neighboring functional groups. This information can only be obtained from ab initio molecular dynamics (MD) calculations such as those based on time-dependent density functional theory (TDDFT) methods [6], which have already been applied to investigate the radiolysis of bulk water [7] and water clusters [8]. In this work, we apply TDDFT MD and report new experimental results to investigate the fragmentation of doubly ionized uracil created in collisions with $100 \mathrm{keV}$ protons. We show not only that theoretical results are compatible with the experimental ones, but also that fragmentation depends critically on the energy, shape, and intramolecular chemical environment of the ionized MO. This suggests that, by targeting specific MOs, damage can be induced rather selectively.

The choice of uracil (a RNA base) follows a rich tradition in experimental collision physics [3,5,9-13]. Uracil was the first biomolecule to be investigated in this context and is often considered as a benchmark due to its simplicity 
and high stability in gas phase (which prevents, e.g., its thermal degradation). The study of uracil in gas phase is also important to understand the early presence of RNA bases on Earth (e.g., the fact that uracil can be formed in outer space suggests an exogenous origin of life [14]). The most detailed experiments on uracil have been performed with proton projectiles of $20-150 \mathrm{keV}[5,9,13]$. In particular, Tabet et al. [5] have reported relative cross sections for electron capture and direct ionization, and the corresponding fragmentation branching ratios. Therefore, $\mathrm{H}^{+} /$uracil collisions are ideal (i) to check the accuracy of existing theoretical methods and (ii) to obtain, with the help of the latter, dynamical information that experiments alone cannot provide.

The experimental setup used in this work has been described in detail elsewhere [15]. Briefly, a pulsed proton beam of $100 \mathrm{keV}$ and $2 \mathrm{~mm}$ diameter collides an effusive uracil gas jet produced by heating commercial powder purchased at Sigma-Aldrich at a temperature of $120^{\circ} \mathrm{C}-150{ }^{\circ} \mathrm{C}$. Charged fragments produced in the collision are mass-over-charge $(M / Z)$ analyzed by means of a time-of-flight (TOF) mass spectrometer operating in second order space focusing with pulsed extraction. The fragments are detected by a high efficiency multichannel plate (MCP) assembled with a secondary electron repeller grid in front of the first MCP. The TOF of all fragments created by one ion pulse is measured by a multistop time device and stored in an event-by-event mode. The TOF is derived from $T=T_{0}-p /(q E)$, where $T_{0}$ is proportional to $\sqrt{M / Z}, p$ is the projection of the momentum along the cell axis, and $E$ is the extraction field.

To theoretically describe the fragmentation of doubly charged uracil, $\mathrm{Ura}^{2+}$, we take advantage of the fact that the collision is very fast and, therefore, that electron removal from the neutral molecule is sudden compared to the fragmentation time. Thus, one can safely assume that the geometry of $\mathrm{Ura}^{2+}$ and the velocities of its nuclei just after the collision are the same as for neutral uracil. We consider two-electron removal from the lowest inner orbitals obtained in the Kohn-Sham (KS) pseudopotential representation of neutral uracil described below, namely, the $9 a^{\prime}$, $10 a^{\prime}$, and $11 a^{\prime}$ orbitals (hereafter called KS1, KS2, and $\mathrm{KS} 3$ ), from the intermediate-energy orbital $17 a^{\prime}$ (KS9), and from the valence highest occupied molecular orbital $5 a^{\prime \prime}$ (HOMO). This orbital sampling allows us to investigate the role of the energy and shape of the ionized $\mathrm{MO}$ on the fragmentation processes, as well as the effect of the bond chemical environment. To account for nonadiabatic effects in the fragmentation arising from inner-shell vacancies, we have performed first-principles calculations within the so-called Ehrenfest MD approximation where the mean field potential energy surface driving the nuclear dynamics is computed at the TDDFT level. Because of the very small time step (of the order of attoseconds) used in the real time propagation of electrons, the total simulation time is necessarily limited to less than $100 \mathrm{fs}$. Since the complete dissociation of the ionized molecule requires in general longer times (a few ps), we then switch to ground-state Born-Oppenheimer (BO) MD when this dynamics becomes essentially adiabatic (i.e., when Ehrenfest and BO MD produce the same trajectory). Fragmentation associated with HOMO vacancies is studied by using standard Car-Parrinello (CP) MD from the very first instant after ionization and thermal equilibration (in this case, fragmentation is so slow-see below-that TDDFT MD is prohibitively expensive).

A detailed description of our TDDFT MD method can be found in Ref. [16]. Briefly, we use a cubic box of size $L=19 \AA$ and a plane wave basis with an energy cutoff of 70 Ry. Core electrons are replaced by pseudopotentials of the standard Troullier-Martins form [17]. The exchange correlation energy is calculated using the generalized gradient approximation functional Becke-Lee-Yang-Parr (BLYP) $[18,19]$. In TDDFT-based Ehrenfest MD, the electronic density evolves according to the time-dependent Kohn-Sham (TDKS) equations [6]. The propagation of the electronic degrees of freedom is started from a nonequilibrium electronic structure in which two electrons are removed from a previously occupied KS orbital of neutral uracil [20]. This initial electron density, which does not correspond to a specific state of $\mathrm{Ura}^{2+}$, is then propagated by numerical integration of the TDKS equations using an iterative Crank-Nicholson algorithm with a time step $\delta t=0.00024$ fs combined with a two-step Runge-Kutta scheme to maintain $\delta t^{3}$ order accuracy. The small step chosen assures energy conservation within $0.02 \%$ $(0.4 \mathrm{eV})$. In the $\mathrm{BO} \mathrm{MD}$ calculations we use $\delta t=$ $0.024 \mathrm{fs}$. The initial atomic configuration is taken from an equilibrated trajectory obtained from a CP MD simulation of the uncharged system at $350 \mathrm{~K}$. The forces on the nuclei are computed according to the Hellman-Feynman theorem as the analytic derivative of the expectation value of the energy. Atomic charges along the different trajectories are evaluated using Bader's atoms-in-molecules approach as implemented in [21].

Fragmentation from vacancies in the HOMO occurs in the ground state of $\mathrm{Ura}^{2+}$ and, consequently, takes much longer. Indeed, no fragmentation is observed in $350 \mathrm{~K} \mathrm{CP}$ MD simulations up to 5 ps due to the presence of dissociation barriers. To allow for an efficient crossing of these barriers, we have performed calculations at 2300 and $2800 \mathrm{~K}$. Fragmentation is the same for both temperatures and occurs now in $\sim 0.5$ ps.

Figure 1 shows the time-of-flight spectrum of singly charged fragments and of two singly charged fragments measured in coincidence. Apart from the obvious difference in intensity, both spectra exhibit similar trends, with pronounced peaks for $M / Z$ (in amu) $\simeq 28,42$, and 69 . The spectrum for noncoincident singly charged fragments is very similar to that obtained with less energetic projectiles $[22,23]$. This suggests that bond breakage is only efficient at specific locations within the molecule. Figure 2 shows 


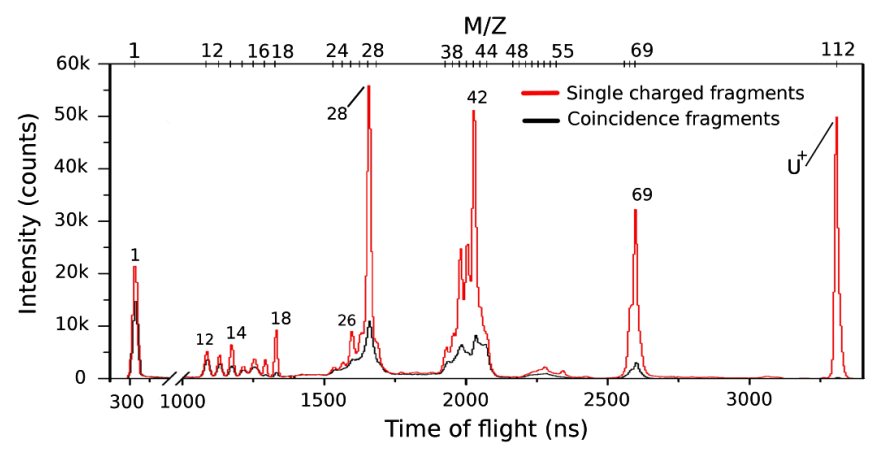

FIG. 1 (color online). Time-of-flight spectra of singly charged uracil fragments (red line) compared to that of charged fragments measured in coincidence (black line). The peak at 112 amu corresponds to uracil ${ }^{+}$.

the 2D ion-ion coincidence spectrum, which is mainly the signature of $\mathrm{Ura}^{2+}$ fragmentation. Only a few channels are visible. Coincidences for $M / Z \leq 16$ (not shown in Fig. 2) are also observed, but they are much less intense (except for $\mathrm{H}^{+}$correlations).

The results of the theoretical calculations are summarized in Fig. 3. The energies of the KS1, KS2, KS3, KS9, and HOMO orbitals, from which two electrons have been removed, are, respectively, $-27,-26,-24,-14$, and $-6 \mathrm{eV} . \mathrm{KS} 1$ and KS2 orbitals are associated with localized $\sigma$-like $\mathrm{CO}$ bonds in a different chemical environment: while $\mathrm{KS} 1$ lies between two $\mathrm{N}$ atoms, KS2 lies between $\mathrm{N}$ and $\mathrm{C}$ atoms. $\mathrm{KS} 3$ is delocalized over one of the $\mathrm{CO}$ bonds and part of the uracil ring. The KS9 and HOMO are even more delocalized since they extend over more than half of the ring. Figure 3 also shows the fragments obtained after removal of two electrons from the above orbitals and their corresponding mass and charge at the end of the dynamics. The values of the calculated charges must be

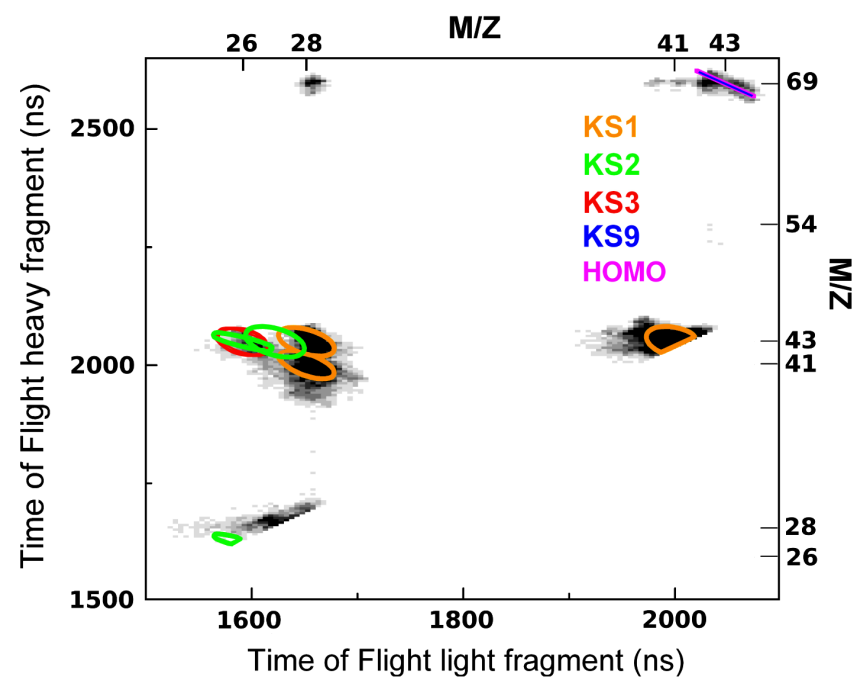

FIG. 2 (color online). 2D TOF coincidence spectrum of charged uracil fragments for $M / Z \geq 22$. Theoretical results for $\mathrm{KS} 1, \mathrm{KS} 2, \mathrm{KS} 3, \mathrm{KS}$, and HOMO fragmentations are represented by open symbols. taken with some caution, since, as is well known, DFT methods can lead to unphysical fractional charges. A paradigmatic example is $\mathrm{H}_{2}^{+}$, for which TDDFT predicts dissociation into $\mathrm{H}^{+0.5}+\mathrm{H}^{+0.5}$, instead of into either $\mathrm{H}+\mathrm{H}^{+}$ or $\mathrm{H}^{+}+\mathrm{H}$. Thus, in general, calculated fractional charges of the order of +0.5 are an indication of the existence of two dissociation paths in which the charge is asymmetrically and alternatively distributed between two fragments.

Removal of two electrons from the KS1 orbital leads to the three fragments shown in Fig. 3. The fragment with mass 41 has a charge of about +1 during the whole trajectory. The rest of the charge is shared between fragments with mass 28 and 43, which arise from the fragmentation of a larger fragment with mass 71 and charge +1 at $\sim 30$ fs. The latter two fragments have charges close to +0.5 , which suggests the formation of a singly charged fragment with mass 28 and a neutral fragment with mass 43 , and the other way around. Therefore, KS1 fragmentation is compatible with the $28 / 41$ and $41 / 43$ coincidences in Fig. 2 (notation: light-fragment-mass/heavy-fragmentmass). It might also be compatible with a $28 / 43$ coincidence. As can be seen, the original CO bond from which the electrons were removed is not broken. This is not the case of KS2 fragmentation: the ionized CO bond breaks at the early stages of the dynamics $(\sim 6 \mathrm{fs})$ leading to

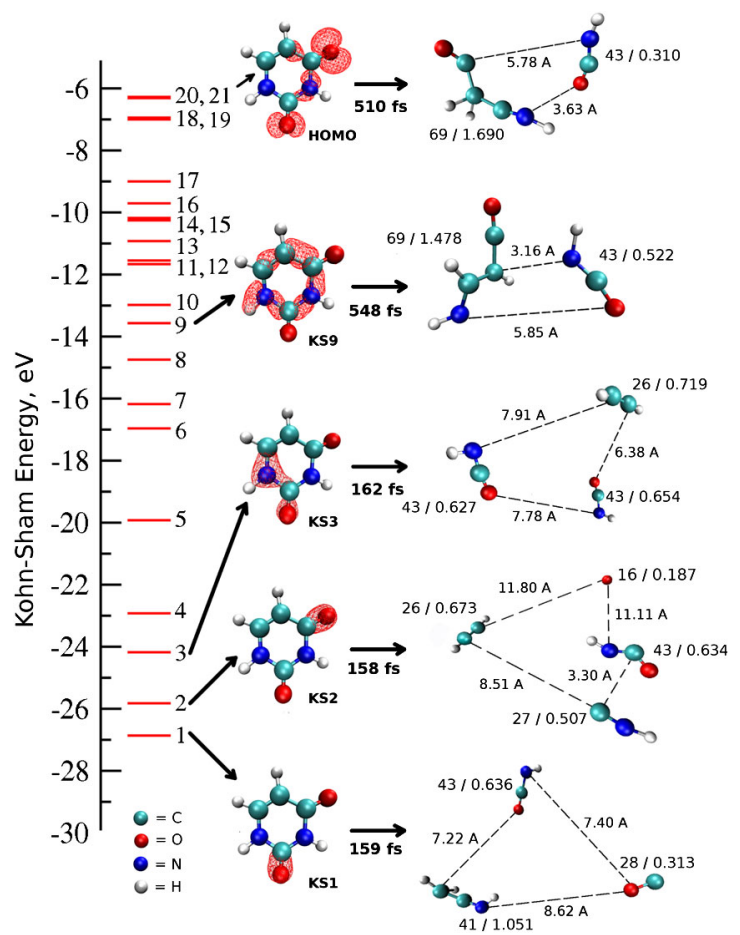

FIG. 3 (color online). Fragmentation resulting from twoelectron removal from the HOMO, KS9, KS3, KS2, and KS1 orbitals (top to bottom). Left: Energies of the occupied KohnSham orbitals of neutral uracil. Middle: Electron densities associated with those orbitals (0.018 isovalue). Right: Fragments observed at the end of the TDDFT $+\mathrm{BO}$ MD propagation, and their corresponding $M / Z$ ratios; fragment-fragment distances (dashed lines) are given in $\AA$. 
ejection of a neutral $\mathrm{O}$ atom; in turn, the $\mathrm{O}$ atom of the other $\mathrm{CO}$ bond migrates from its initial position to form a new $\mathrm{CO}$ bond with the neighboring $\mathrm{C}$ atom that has just lost its $\mathrm{O}$ atom. As a result, fragments with mass 26, 27, and 43, and charge of the order of +0.5 , are produced. Thus, KS2 fragmentation is compatible with coincidence signals at 26/27, 26/43, and 27/43 (see Fig. 2). None of these coincidences are seen in KS1 fragmentation, showing that chemical environment plays a crucial role. Removal of two electrons from KS3 leads to a charged fragment with mass 26 plus two charged fragments with mass 43 , thus leading to a 26/43 coincidence. KS9 and HOMO fragmentation leads to a 43/69 coincidence (see Fig. 2), which is likely the case for two-electron vacancies created in any orbital between them. Notice that the energetically most favorable dissociation path, $\mathrm{HNCH}_{2} \mathrm{CO}^{+}+$ $\mathrm{OCNH}^{+}$, is only seen in fragmentation from the higher orbitals (see Ref. [24] for the relative energies of the different fragmentation channels). Additional calculations in which the internal energy is comparable to that available in vacancies created in the KS6-KS7 orbitals lead to very similar fragments, and the corresponding coincidences are compatible with experiment (see Ref. [24]).

Combining the above information with the calculated velocities for each fragment, we have generated the $2 \mathrm{D}$ TOF spectrum that is superimposed to the experimental one in Fig. 2. As can be seen, the calculations reproduce the most intense coincidence signals, as well as (in most cases) their shape. This suggests that not only the fragments but also their relative velocities are reasonably described by theory. In any case, the appearance of fractional charges leads inevitably to errors in the calculated kinetic energies; however, we have checked that velocities even twice as large as those resulting from the calculations are still compatible with the experimental findings.

A striking feature in KS1, KS2, and KS3 fragmentation is that charge reorganization and localization on various atomic centers is nearly completed in $\sim 20 \mathrm{fs}$, a short time compared to that required for vibrations and bond breaking. After this, no significant changes in the electron density are observed. Thus, every two-electron vacancy can be associated with the formation of a specific precursor state during the early stages of the dynamics. Since the energy of each precursor state is different, the observed dissociation does not necessarily follow the minimum energy path. In contrast, for fragmentation arising from vacancies in the higher orbitals, the charge is strongly delocalized before dissociation, which leads to a sort of equilibration of the system, thus favoring fragmentation along the energetically most favorable path.

In summary, a combination of TDDFT and BO MD methods shows that two-electron removal from uracil leads to fragments in good agreement with ion-ion coincidence experiments in which $100 \mathrm{keV}$ protons collide with gas-phase uracil. Calculations show that fragmentation from inner-shell vacancies strongly depends on the intramolecular chemical environment and almost never proceeds through the energetically most favorable dissociation path. Besides bond breaking, atom migration leading to the formation of new bonds is also observed. The next step will be to test the validity of these predictions when the biomolecule is embedded in an environment resembling that of living systems.

We thank Mare Nostrum BSC, CCC-UAM, IDRIS, and CINES (Grant No. x2010085014) for computer time. Work supported by the MICINN projects FIS2010-15127, CTQ2010-17006, ACI2008-0777, and CSD 2007-00010, the CAM project S2009/MAT1726, the COST Action CM0702, the Picasso project HF2007-0067, and HPC Europa2. P.L.-T. thanks the University of Evry for its hospitality.

*fernando.martin@uam.es

[1] E. Fokas et al., Biochim. Biophys. Acta 1796, 216 (2009).

[2] Z. Deng et al., Phys. Rev. Lett. 95, 153201 (2005).

[3] J. de Vries et al., Phys. Rev. Lett. 91, 053401 (2003).

[4] B. Liu et al., Phys. Rev. Lett. 97, 133401 (2006).

[5] J. Tabet et al., Phys. Rev. A 81, 012711 (2010).

[6] E. Runge and E. K. U. Gross, Phys. Rev. Lett. 52, 997 (1984).

[7] I. Tavernelli et al., Chem. Phys. Chem. 9, 2099 (2008).

[8] U.F. Ndongmouo-Taffoti et al., Eur. Phys. J. D 58, 131 (2010).

[9] B. Coupier et al., Eur. Phys. J. D 20, 459 (2002).

[10] J. de Vries et al., J. Phys. B 35, 4373 (2002).

[11] J. de Vries, R. Hoekstra, R. Morgenstern, and T. Schlathoelter, Phys. Scr. T110, 336 (2004).

[12] T. Schlatholter, R. Hoekstra, and R. Morgenstern, Int. J. Mass Spectrom. 233, 173 (2004).

[13] P. Moretto-Capelle and A. Le Padellec, Phys. Rev. A 74, 062705 (2006).

[14] M. Nuevo et al., Astrobiology 9, 683 (2009).

[15] P. Cafarelli et al., AIP Conf. Proc. 1080, 71 (2008).

[16] I. Tavernelli, U.F. Rohrig, and U. Rothlisberger, Mol. Phys. 103, 963 (2005).

[17] N. Troullier and J.L. Martins, Phys. Rev. B 43, 1993 (1991).

[18] A. D. Becke, Phys. Rev. A 38, 3098 (1988).

[19] C. Lee, W. Yang, and R. G. Parr, Phys. Rev. B 37, 785 (1988).

[20] Electron removal from different MOs is not considered because spin-polarized calculations are prohibitive.

[21] W. Tang, E. Sanville, and G. Henkelman, J. Phys. Condens. Matter 21, 084204 (2009).

[22] S. Denifl et al., Int. J. Mass Spectrom. 238, 47 (2004).

[23] H.-W. Jochims et al., Chem. Phys. 314, 263 (2005).

[24] See Supplemental Material at http://link.aps.org/ supplemental/10.1103/PhysRevLett.107.023202 for relative energies of the different fragmentation channels in $\mathrm{Ura}^{2+}$, additional fragmentation conditions, and stability of the results with respect to errors in the calculated fragment velocities. 